\title{
Primeira descrição epidemiológica da leishmaniose visceral em Campo Grande, Estado de Mato Grosso do Sul
}

\author{
First epidemiological description of visceral leishmaniasis \\ in Campo Grande, State of Mato Grosso do Sul
}

Andrea Cristina Alpoim Botelho ${ }^{1}$ e Delsio Natal ${ }^{2}$

\begin{abstract}
RESUMO
Foi realizado estudo epidemiológico sobre casos de leishmaniose visceral notificados em Campo Grande de 2001 a 2006, utilizando-se dados do Sistema de Informação de Agravos de Notificação. Foram registradas 577 notificações com incidência de 1,47 casos/100.000hab em 2001, chegando a 20,98 casos $/ 100.000$ hab em 2006, com notificações todos os meses a partir de 2002. Crianças até nove anos contribuíram com $40 \%$ dos casos. 0 sexo masculino contribuiu com $64 \%$ das notificações e o sexo feminino com $36 \%$. A letalidade variou de $5 \%$ a $11 \%$, com média de $8 \%$. Dos 44 óbitos, 33 (75\%) ocorreram no sexo masculino e 11 (25\%) no sexo feminino. Embora os idosos tenham contribuído com 9\% dos casos, a mortalidade entre eles alcançou 39\%. Foram 27 casos de co-infecção Leishmania/HIV (5\%) com letalidade de 15\%, a maioria em homens dos 20 aos 49 anos. Constatou-se processo de endemização da doença com elevada incidência.
\end{abstract}

Palavras-chaves: Leishmaniose visceral. Epidemiologia. Saúde Pública. Co-infecção Leishmania/HIV. Urbanização.

\begin{abstract}
An epidemiological study was conducted on cases of visceral leishmaniasis that were notified in Campo Grande between 2001 and 2006, using data from the Brazilian information system for notifiable diseases (Sistema de Informação de Agravos de Notificação, SINAN). In 2001, 577 cases were notified, giving an incidence of 1.47 cases/100,000. This rose to 20.98 cases/100,000 in 2006. From 2002 onwards, new cases were notified every month. Children up to nine years of age accounted for $40 \%$ of the cases. Males accounted for $64 \%$ of the cases and females, $36 \%$. The death rate ranged from 5 to $11 \%$, with a mean of $8 \%$. Among the 44 deaths, 33 (75\%) were male cases and 11 (25\%) were female cases. Although elderly people accounted for $9 \%$ of the cases, mortality among them reached 39\%. There were 27 cases of Leishmania /HIV coinfection (5\%), with a mortality rate of $15 \%$, mostly among men aged 20 to 49 years. A process of disease endemization with a high rate of incidence was observed.
\end{abstract}

Key-words: Visceral leishmaniasis. Epidemiology. Public Health. Leishmania/HIV coinfection. Urbanization.

A leishmaniose visceral é uma doença emergente e negligenciada ${ }^{31}$ que apresenta ampla distribuição em países de clima tropical e subtropical ${ }^{12}$. Estima-se que anualmente ocorram cerca de 500 mil casos novos da doença. Brasil, Bangladesh, Índia e Sudão contribuem com $90 \%$ dos $\operatorname{casos}^{31}$. 0 agente etiológico da leishmaniose visceral no Brasil é o protozoário

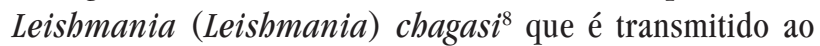
homem por meio da picada de flebotomíneos fêmeas da espécie Lutzomyia longipalpis $^{29}$. 0 protozoário provoca uma doença crônica e sistêmica com intenso parasitismo das células do sistema fagocítico mononuclear ${ }^{3}$. Baço, fígado e medula óssea são particularmente acometidos e a forma clássica da doença é

1.Curso de Pós-Graduação em Saúde Pública/Epidemiologia. Faculdade de Saúde Pública. Universidade de São Paulo, São Paulo, SP. 2.Departamento de Epidemiologia. Faculdade de Saúde Pública. Universidade de São Paulo, São Paulo, SP.

Endereço para correspondência: Dr. Delsio Natal. Dept ${ }^{\underline{0}}$ de Epidemiologia/FSP/USP. Av. Dr. Arnaldo 715, Cerqueira César, 01246-904 São Paulo, SP.

Tel: 5511 3066-7714; Fax: 5511 3081-2108.

e-mail: andreabotelho@usp.br; natal@usp.br

Recebido para publicação em 07/01/2009

Aceito em 02/09/2009 descrita pela tríade: febre, hepatoesplenomegalia e pancitopenia. Cerca de $90 \%$ dos casos são fatais se a pessoa não receber tratamento, sendo que hemorragias e infecção generalizada são as causas da morte pela doença ${ }^{17}{ }^{18}$. Com o surgimento da AIDS, a leishmaniose ganhou importância devido à suscetibilidade dos portadores do HIV ${ }^{1732}$.

A leishmaniose visceral foi reconhecida no Brasil em razão das pesquisas feitas por Evandro Chagas e equipe na segunda metade da década de 1930 que assinalaram a doença como Nova entidade mórbida do homem da América do Sul e por Deane em 1956 que a descreveu como endêmica no Ceará9. Até o final do século $\mathrm{XX}$, a doença acometia pessoas de áreas rurais, principalmente do interior do Nordeste que contribuía com 90\% dos casos no Brasil. Depois disso, houve mudança no padrão de transmissão da doença que passou a ser predominantemente urbano, primeiramente nas periferias das capitais dos estados nordestinos e depois, como doença pouco conhecida, se dispersou para outras cidades e regiões do país onde nunca havia alcançado ${ }^{17}$.

Atualmente, a leishmaniose visceral é notificada em todos os estado da federação, exceto no Acre. No período 2001 a 2006, 
os estados que apresentaram o maior número de casos foram Maranhão (3.562 casos), Minas Gerais (2.512 casos), Ceará (2.431 casos), Pará (1.970 casos) e Mato Grosso do Sul (1.189 casos $)^{20}$. Entre as capitais, já foram descritas epidemias em Teresina de 1981 a 1985, que voltou a ocorrer em 1992 e 1994. Em São Luis houve uma epidemia de 1982 a 1986 que voltou no biênio 1993-199430. Em Natal, casos foram registrados de 1991 a $1992^{15}$. Na região metropolitana de Belo Horizonte, foram notificados 345 casos autóctones de 1994 a $1999^{24}$. A doença também avançou para outros estados e regiões em direção sul e em 2006 foi registrado 0 primeiro caso autóctone na Argentina ${ }^{26}$.

Campo Grande apresenta uma situação peculiar, pois, dos 1.189 casos registrados no estado de 2001 a 2006, 577 (49\%) ocorreram na capital ${ }^{20}$. De 1999 a 2000, foram realizadas as primeiras capturas de flebotomíneos em Campo Grande. Dos 1.245 exemplares capturados, apenas 71 eram da espécie Lutzomyia longipalpis ainda sem a ocorrência de casos humanos ${ }^{22}$. Outras capturas foram feitas em 2004 e 2005 e dos 5.004 exemplares, 4.615 eram Lutzomyia longipalpis ${ }^{23}$, apontando a adaptação do vetor ao ambiente urbano e coincidindo com a ocorrência de casos humanos e caninos na cidade. De maio de 2003 a abril de 2005, foram coletados nos domicílios e peridomicílios 2.275 desses dípteros, sendo que 2.115 eram Lutzomyia longipalpi s $^{27}$. Atualmente, a leishmaniose visceral é um importante problema de saúde pública em Campo Grande, com a notificação crescente de casos e óbitos. Considerando a atual importância da leishmaniose visceral nessa cidade e a inexistência de um estudo epidemiológico descritivo, faz-se necessário pesquisar as características deste agravo no município.

\section{MATERIAL E MÉTODOS}

Área de estudo. Campo Grande é a capital do estado de Mato Grosso do Sul, com 8.096 kilômetros quadrados, ocupando $2 \%$ do território dessa Unidade da Federação ${ }^{14}$. A população era de 724.524 habitantes em 2007, o que corresponde a $32 \%$ da população do estado ${ }^{19}$. 0 clima é do tipo tropical de savana, caracterizado por período seco no outono e inverno e chuvoso nos meses de primavera e verão. A temperatura média anual é de $23^{\circ} \mathrm{C}^{14}$. A cidade é marcadamente horizontalizada e possui um programa para evitar a proliferação de favelas. Os acampamentos na periferia são transformados em bairros urbanizados com casas populares em loteamentos com asfalto, calçamento, energia elétrica e encanamento de água e esgoto. Aos poucos são perdidas as características de loteamento pioneiro, transformando-se em um bairro residencial comum. Nesses locais existem escolas, pequenos comércios e postos de atenção básica de saúde, sendo que alguns funcionam até as 22 horas. A zona sul é chamada Anhanduizinho que reproduz as características descritas anteriormente.

Fonte de dados. Os dados foram obtidos por meio do acesso ao banco do Sistema de Informação de Agravos de Notificação do Ministério da Saúde, disponível na internet. 0 período de estudo foi de 2001 a 2006 e foram selecionados todos os casos e óbitos por leishmaniose visceral segundo o município de residência.
Dados sobre a população de Campo Grande foram obtidos no banco de dados da Rede Interagencial de Informações para a Saúde na internet, que tem como base o censo, contagens populacionais e as projeções demográficas do Instituto Brasileiro de Geografia e Estatística. Dados sobre os casos e óbitos por coinfecção Leishmania/HIV foram obtidos por meio de contato com a Gerência Técnica de Leishmanioses do Ministério da Saúde em Brasília.

Análise dos dados. Utilizou-se o método epidemiológico descritivo que tem como base as variáveis relacionadas à pessoa, lugar e tempo ${ }^{21}$. Por se tratar de um agravo infeccioso e com base nos casos novos e confirmados, foi calculado o coeficiente de incidência da doença por 100.000 habitantes, dividindo-se 0 número de casos pelo total da população residente no mesmo período e em seguida multiplicando-se o resultado por 100.000 . No caso de grupo etário específico, o cálculo foi feito dividindose o número de casos ocorridos em determinada faixa etária pela população da mesma idade e ano. 0 mesmo foi feito para o cálculo de incidência por sexo. 0 coeficiente de letalidade foi calculado dividindo-se o número de óbitos pelo número de casos e em seguida multiplicando-se o resultado por 100. Em relação ao lugar, a única variável disponível no banco de dados foi o próprio município de Campo Grande e por fim, as variáveis relativas ao tempo foram os anos que ora são apresentados de forma acumulada no período 2001 a 2006, ora são apresentados em separado ano a ano. Para a tabulação dos dados e montagem das figuras e tabelas utilizou-se o programa Excel.

\section{RESULTADOS}

Informações epidemiológicas gerais. A leishmaniose visceral em Campo Grande apresentou tendência de expansão, com aumento no número de casos a partir de 2002. Foram 577 notificações no período sendo que, em média, foram registrados 96 casos por ano o que corresponde a oito casos por mês. A partir de 2002 ocorreram registros em todos os meses do ano sem padrão regular de sazonalidade. 0 número de casos em cada ano foi: 10 casos em 2001, 22 casos em 2002, 97 casos em 2003, 130 casos em 2004, 157 casos em 2005 e 161 casos em 2006.

A incidência por 100.000 habitantes mostrou o mesmo comportamento ascendente. Nos últimos dois anos nota-se tendência de estabilização, porém com valor alto, sem sinal de diminuição do coeficiente de incidência (Figura 1).

A maioria dos doentes residia em área urbana. A partir de 2003, não foram registrados casos em área rural. A proporção de casos segundo zona de residência, considerando o período acumulado foi: 99\% dos casos em área urbana, 0,3\% em área rural, 0,2\% em área urbana/rural e 0,5\% em área desconhecida.

Dos 577 casos notificados, 460 (80\%) se submeteram ao exame parasitológico para diagnóstico (Tabela 1). Apenas 88 (15\%) pessoas não realizaram o exame. Em 29 (5\%) casos não se sabe se a pessoa realizou a prova parasitológica. Dos 460 exames parasitológicos realizados, 80 (17\%) mostrou resultado negativo para Leishmania spp. 


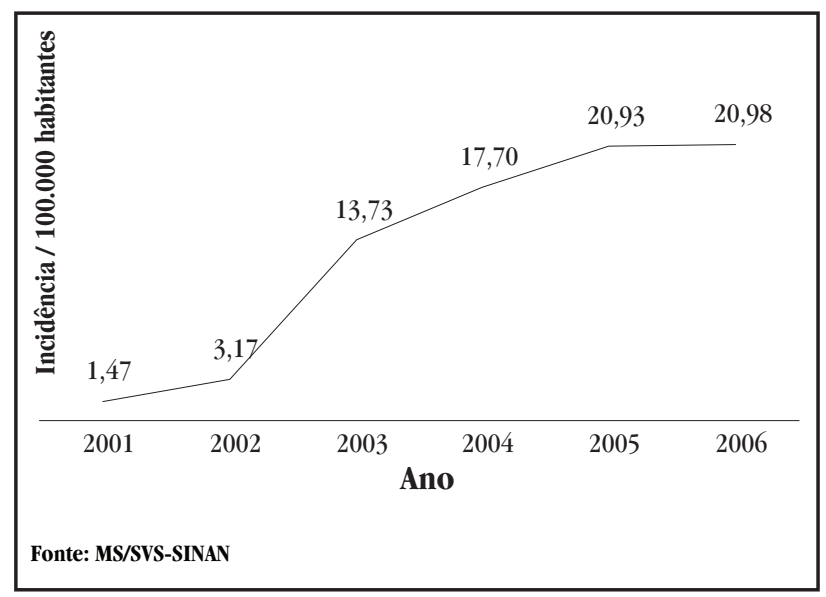

FIGURA 1

Coeficiente de incidência de leishmaniose visceral (por 100.000 habitantes), segundo ano. Campo Grande, MS, 2001 a 2006.

\section{TABELA 1}

Resultado do exame parasitológico para leishmaniose visceral, segundo ano de diagnóstico. Campo Grande, MS, 2001 a 2006.

\begin{tabular}{lrrrrrrr}
\hline & \multicolumn{7}{c}{ Ano } \\
\cline { 2 - 6 } Resultado & 2001 & 2002 & 2003 & 2004 & 2005 & 2006 & Total \\
\hline Positivo & 4 & 12 & 74 & 93 & 97 & 100 & $\mathbf{3 8 0}$ \\
Negativo & 1 & 2 & 9 & 16 & 15 & 37 & $\mathbf{8 0}$ \\
\hline Total & 5 & 14 & 83 & 109 & 112 & 137 & 460 \\
\hline
\end{tabular}

Fonte: MS/SVS-SINAN
Informações referentes à pessoa. Calculando o coeficiente de incidência segundo sexo é possível observar que o risco de adoecer por leishmaniose visceral é maior no sexo masculino. Em relação à idade e gênero, a diferença não é bem clara nas faixas etárias de um a quatro anos e de dez a 19 anos, idades em que ambos os sexos parecem ser igualmente vulneráveis. A diferença entre gêneros e idade é mais notada a partir dos 20 anos. Na vida adulta, os homens são mais acometidos. De maneira geral, os extremos da vida apresentaram maior risco, embora as demais faixas etárias também tenham apresentado risco elevado (Figura 2). Em relação à proporção, os indivíduos masculinos contribuíram com 369 dos 577 casos, perfazendo 64\% das notificações. 0 sexo feminino contribuiu com 213 dos 577 casos, o que corresponde a 36\% das notificações. Dos 577 casos, 233 (40\%) acometeram crianças até nove anos de idade.

Informações sobre os óbitos. Ocorreram 44 óbitos pela doença, com tendência de redução na série temporal. 0 coeficiente de letalidade em cada ano foi: $10 \%$ em 2001, $9 \%$ em 2002, 11\% em 2003, 5\% em 2004, 8\% em 2005 e 7\% em 2006. No período acumulado, a letalidade foi de $8 \%$. Em relação à mortalidade segundo sexo, dos 44 óbitos, 33 (75\%) ocorreram no sexo masculino e 11 (25\%) no sexo feminino. 0 coeficiente de letalidade foi de $11 \%$ no sexo masculino e $2 \%$ no sexo feminino. As pessoas a partir dos 40 anos apresentaram maior risco de morrer em decorrência da doença. Embora as crianças tenham apresentado as maiores incidências, a mortalidade entre elas foi pouco expressiva. Destaca-se a alta mortalidade entre os idosos. Apesar do número de casos entre idosos ter sido pequeno, tornando a proporção de óbitos muito elevada, é possível observar que o risco de morrer aumenta conforme a idade. Por outro lado, a mortalidade foi zero em jovens de 15 a 19 anos (Figura 3).

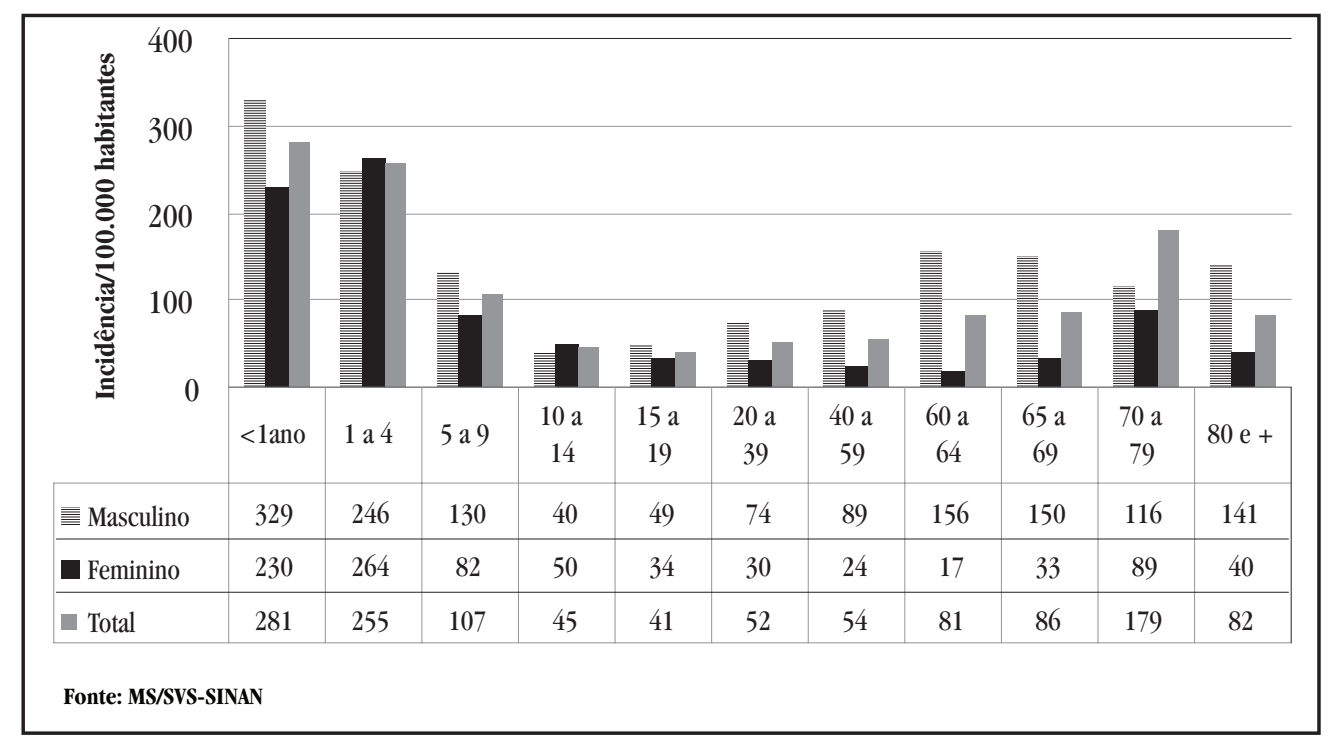

FIGURA 2

Coeficiente de incidência de leishmaniose visceral (por 100.000 habitantes), segundo sexo e faixa etária. Campo Grande, MS, 2001 a 2006. 


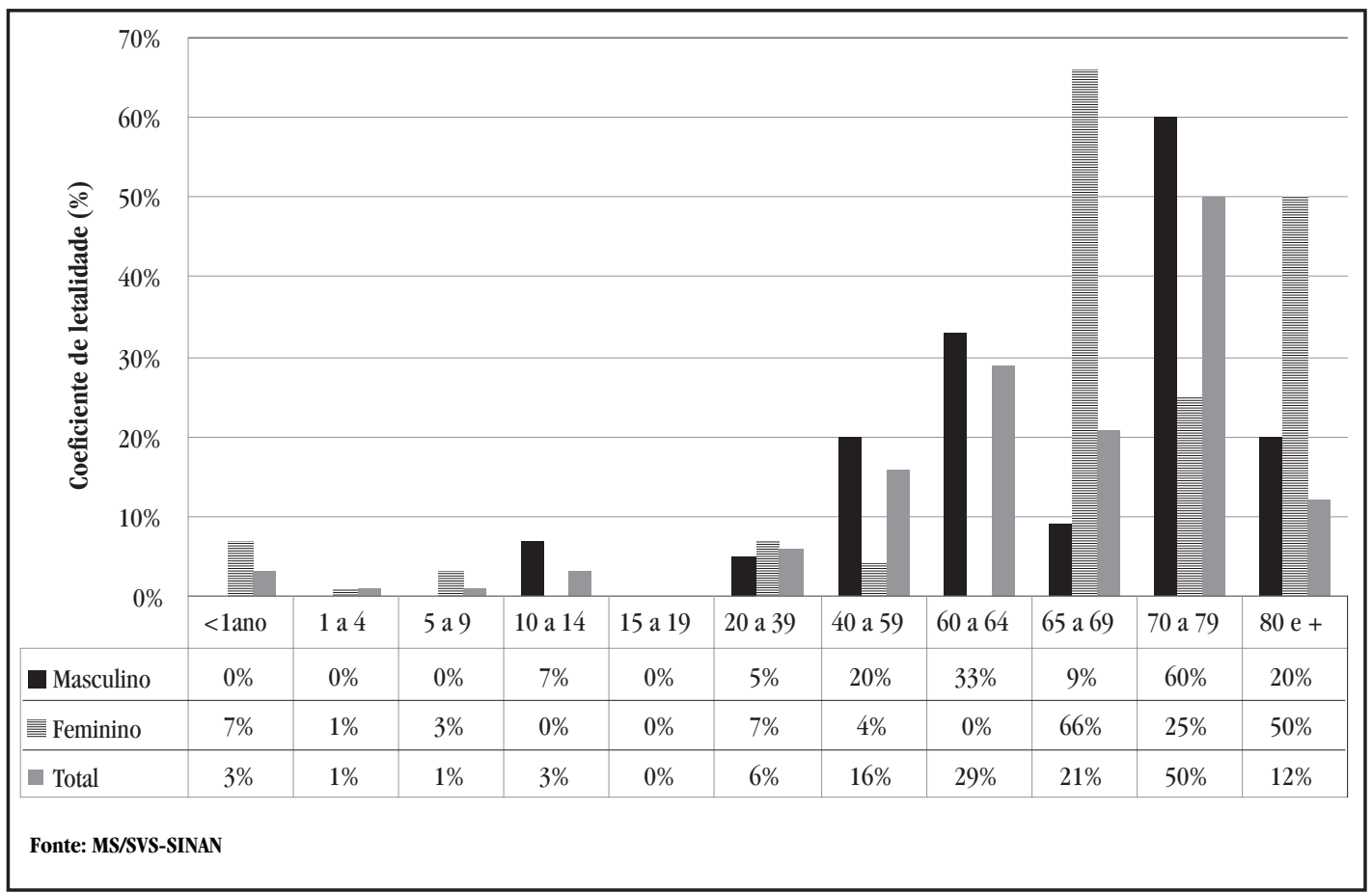

FIGURA 3

Coeficiente de letalidade por leishmaniose visceral (\%), segundo sexo e faixa etária. Campo Grande, MS, 2001 a 2006.

Informações sobre a co-infecção Leishmania/HIV. Foram notificados 27 casos de co-infecção Leishmania/HIV, detectados a partir de 2002, representando 5\% do total de casos de leishmaniose visceral notificados. 0 sexo masculino contribuiu com 21 dos 27 casos de co-infecção, perfazendo $78 \%$ dos registros e apenas seis casos de co-infecção ocorreram no sexo feminino, 0 que corresponde a $22 \%$ dos casos de Leishmania/HIV, apontando tendência de masculinização semelhante à observada nos casos de leishmaniose visceral isolada. De 2002 a 2006 ocorreram quatro óbitos por co-infecção Leishmania/HIV, sendo três em homens e apenas um em mulher. A letalidade foi de $15 \%$. Todos os óbitos ocorreram na faixa etária dos 20 aos 49 anos.

\section{DISCUSSÃo}

Este trabalho é a primeira descrição epidemiológica da leishmaniose visceral no município de Campo Grande. Os resultados mostram que no período de 2001 a 2006 a doença apresentou característica de expansão, principalmente a partir de 2002 e até o final do período analisado não houve sinal de diminuição no número de casos. Ao contrário, comparandose 2001 com 2006, houve aumento de $1510 \%$ no número de notificações. Em relação à sazonalidade, não foi possível observar um padrão regular de notificações da doença. De qualquer forma, foi possível notar que a atividade de Lutzomyia longipalpis é intensa e está presente 0 ano todo, refletindo em notificações em todos os meses a partir de 2002, ano que coincide com a explosão de casos no município. Apesar do Programa Nacional de Controle da Leishmaniose Visceral recomendar a aplicação de inseticidas logo após o período chuvoso ${ }^{17}$, esta estratégia parece não ser útil para controlar o flebotomíneo em Campo Grande.
Em 2001, a incidência da doença foi de 1,47 casos para cada 100.000 habitantes, chegando a 20,98 casos para cada 100.000 habitantes, sendo a maior incidência do país entre as capitais dos estados no ano 2006, seguido por Teresina (13,84 casos/100.000 habitantes), Palmas (13,58 casos/100.000 habitantes), Fortaleza (7,90 casos/100.000 habitantes) e Belo Horizonte $(5,47$ casos/100.000 habitantes) ${ }^{20}$.

0 diagnóstico é importante para conduzir o tratamento adequado da doença. 0 exame parasitológico é a prova definitiva, pois detecta os parasitos nos tecidos humanos ${ }^{11}$. Os resultados mostraram que 460 pessoas se submeteram a esse exame e que destas, 80 (17\%) apresentaram resultado negativo para Leishmania spp. Como tratar uma doença que causa todas as características clínicas da leishmaniose visceral, mas não se consegue provar a existência dos protozoários nos tecidos humanos? Essa questão é complexa e pode sugerir que outras espécies de Leishmania podem estar causando manifestações clínicas compatíveis com a leishmaniose visceral. Estudo publicado em 1986 relatou o caso de um paciente com manifestações clínicas clássicas da leishmaniose visceral sem a presença do protozoário no baço e fígado apesar destes estarem com as dimensões aumentadas. Foram detectadas amastigotas de Leishmania (Leishmania) amazonensis na medula óssea ${ }^{4}$. Outro estudo mostrou que de 46 casos de leishmaniose visceral, 11 tiveram como agente causal a Leishmania (Leishmania) amazonensis ${ }^{5}$. Outros trabalhos mostraram resultados semelhantes que detectaram Leishmania (Leishmania) braziliensis na medula óssea ${ }^{13}$ e Leishmania (Leishmania) amazonensis em linfonodo ${ }^{1}$.

Foi observada a tendência de masculinização da doença, com proporção masculino/feminino de aproximadamente 2:1. 
Este resultado é semelhante aos encontrados em Natal ${ }^{15}$, Belo Horizonte $^{28}$, Mato Grosso ${ }^{16}$ e Bahia ${ }^{829}$ e coincide com a literatura especializada ${ }^{3}$. Em relação à faixa etária, foi possível observar que as crianças até nove anos foram mais acometidas, contribuindo com $40 \%$ do total de casos registrados neste estudo, proporção semelhante à encontrada em outros trabalhos 38152829.

0 coeficiente de letalidade no período acumulado foi de $8 \%$, variando de $5 \%$ a $11 \%$, somando 44 óbitos. A letalidade em Campo Grande é um pouco maior que a média nacional no mesmo período que variou de $6 \%$ a $8 \%$. Dos 20.530 casos notificados, no Brasil de 2001 a 2006, 1.459 evoluíram para óbito indicando letalidade de $7 \%$, em média ${ }^{20}$. Já em Belo Horizonte, a letalidade variou de $7 \%$ a $17 \%$ de 1994 a $1999^{29}$. Embora as crianças sejam mais afetadas, o desfecho mais comum entre elas foi a cura, com letalidade variando de $1 \%$ a $3 \%$ na faixa etária de zero a nove anos. Em Recife a letalidade pela doença entre as crianças foi de $10,2 \%^{24}$ e em São Paulo 9,3\% ${ }^{6}$. Uma das explicações para a baixa letalidade entre as crianças em Campo Grande pode ser pelo bom preparo dos serviços de saúde do município. Por outro lado, os idosos apresentaram situação preocupante, pois a letalidade entre eles variou de $12 \%$ a $50 \%$. Apesar dos idosos terem contribuído com apenas 9\% dos casos, os óbitos entre eles alcançaram 39\% do total de mortes pela doença. Uma possível explicação para este fato pode estar relacionada à diminuição da capacidade de resposta do sistema imunológico da pessoa idosa e o conhecimento dos profissionais de saúde que associam a leishmaniose visceral a um agravo predominantemente infantil, diminuindo as suspeitas em idosos. Esse resultado parece ser inédito em trabalhos epidemiológicos sobre a leishmaniose visceral. Por esse motivo, existe a necessidade de mais estudos sobre o comportamento da doença entre os idosos, principalmente no tocante à mortalidade. A melhor situação foi observada entre os jovens de 15 a 19 anos que além de apresentarem a menor incidência da doença também não contribuíram com nenhum óbito no período analisado.

A co-infecção Leishmania/HIV contribuiu com 5\% do total de casos notificados e esse valor é semelhante aos relatos apresentados na Conferência da Organização Mundial de Saúde sobre co-infecção Leishmania/HIV que mostraram que as proporções de casos Leishmania/HIV variam de $2 \%$ a $12 \%$ nos 35 países onde os dois agravos são detectados. Casos extremos são observados na África, onde $30 \%$ dos pacientes com leishmaniose visceral também estão infectados pelo vírus HIV ${ }^{27}$. Os adultos de 20 a 49 anos contribuíram com $81 \%$ dos casos de co-infecção registrados em Campo Grande. No Brasil, em média, 86\% dos casos de co-infecção acometem pessoas de 20 a 49 anos $^{25}$. Proporções semelhantes também foram registradas na Europa ${ }^{10}$.

0 sexo masculino foi mais vulnerável à co-infecção Leishmania/HIV, com 78\% dos casos registrados no período 2002 a 2006. Essa proporção é semelhante à encontrada na Europa que mostrou que $83 \%$ dos casos de co-infecção incidem em pessoas do sexo masculino ${ }^{1032}$. Um estudo feito no Brasil mostrou que o sexo masculino contribuiu com $78 \%$ dos casos de co-infecção no período $2001 \mathrm{a} 2005^{25}$. A maior incidência no sexo masculino pode ser explicada, em parte, pela masculinização da AIDS e da leishmaniose visceral, tornando-os mais vulneráveis. Ocorreram quatro óbitos por co-infecção, sendo três no sexo masculino. 0 coeficiente de mortalidade foi de $15 \%$. Esta proporção é superior à mortalidade observada nos casos de infecção apenas pela Leishmania (Leishmania) chagasi, mas é inferior à mortalidade por co-infecção Leishmania/HIV observada em outros trabalhos ${ }^{25} 32$.

Acredita-se que os números da leishmaniose visceral em Campo Grande sejam maiores do que os que foram apresentados, considerando que existe subnotificação de casos e óbitos. Este trabalho foi baseado em um banco de dados secundário que é alimentado por notificação passiva do agravo, o que dificulta aprofundamento analítico. Apesar das imprecisões, as informações apresentadas são úteis para se conhecer melhor as características da doença no município de Campo Grande que parece enfrentar não uma epidemia de leishmaniose visceral, mas sim um processo de endemização urbana da doença com alta incidência de casos.

\section{AGRADECIMENTOS}

Agradecemos à Waleska Alexandra Alves da Gerência Técnica de Leishmanioses do Ministério da Saúde, por nos fornecer dados sobre a co-infecção Leishmania/HIV.

\section{REFERÊNCIAS}

1. Aleixo JA, Nascimento ET, Monteiro GR, Fernandez MZ, Ramos AMO, Wilson ME. Atypical American visceral leishmaniasis caused by Leishmania amazonensis infection presenting with hepatitis and adenopaty. Transactions of the Royal Society of Tropical Medicine and Hygiene 100:79-82, 2006.

2. Ashford RW. Leishmaniasis. In: Service MW (ed) The encyclopedia of arthropodtransmited infections of man an domesticated animals. London, p. 269-279, 2001.

3. Badaró R, Duarte MIS. Leishmaniose visceral (calazar). In: Veronesi R, Focaccia R (ed) Tratado de infectologia. Editora Atheneu, São Paulo, p.187-268, 1996.

4. Barral A, Badaró R, Barral-Netto M, Grimaldi Jr G, Momem H, Carvalho EM. Isolation of Leishmania mexicana amazonensis from the bone marrow in a case of American visceral leishmaniasis. The American Journal of Tropical Medicine and Hygiene 35:732-734, 1986.

5. Barral A, Pedral-Sampaio D, Grimaldi Jr G, Momen H, McMahon-Pratt D, Jesus AR, Almeida R, Badaró R, Barral-Netto M, Carvalho EM, Johnson Jr WD. Leishmniasis in Bahia, Brazil: Evidence that Leishmania amazonensis produces a wide spectrum of clinical disease. The American Journal of Tropical Medicine and Hygiene 44:536-546, 1991.

6. Campos Jr D. Características do calazar na criança: estudo de 75 casos. Pediatria. 71:261-265, 1995.

7. Chagas E, Cunha AM, Oliveira G, Romaña C. Leishmaniose visceral americana (Nova entidade mórbida do homem da América do Sul). Memórias do Instituto Oswaldo Cruz 2:325-329, 1937.

8. Cunha S, Freire M, Eulálio C, Cristóvão J, Netto E, Johnson WD, Reed SG, Badaró R. Visceral leishmaniasis in a new ecological niche near a major metropolitan area of Brazil. Transactions of the Royal Society of Tropical Medicine and Hygiene. 89:115-158, 1995.

9. Deane LM. Leishmaniose visceral no Brasil. Estudos sobre reservatórios e transmissão realizada no estado do Ceará. Serviço Nacional de Educação Sanitária. Rio de Janeiro, p. 162, 1956. 
10. Desjeux P, Alvar J. Leishmania/HIV co-infection: epidemiology in Europe. Annals of Tropical Medicine and Parasitology. 97:3-15, 2003.

11. Dietze R. Diagnóstico sorológico e parasitológico da leishmaniose visceral. In: Informe final de la reunião de expertos OPS/OMS sobre leishmaniasis visceral em las Américas. Organización Panamericana de La Salud. Rio de Janeiro, p.63-65, 2006.

12. Grimaldi Jr G, Tesh RB, McMahon-Pratt D. A review of the geographic distribuition and epidemiology of leishmaniasis in the new world. The American of Tropical Medicine and Hygiene 41:687-675, 1989.

13. Hernández D, Rodriguez N, Martinez C, García L, Convit J. Leishmania braziliensis causing visceral leishmaniasis in a patient with immunodeficiency virus infection, identified with aid of polymerase chain reaction. Transactions of the Royal Society of Tropical Medicine and Hygiene 87:627-628, 1993.

14. Instituto Brasileiro de Geografia e Estatística. www.ibge.gov.br/cidades/default, Brasília, 2008

15. Jeronimo SMB, Oliveira RM, Mackay S, Costa RM, Sweet J, Nascimento ET, Luz KG, Fernandes MZ, Jernigan J, Pearson RD. An urban outbreak of visceral leishmaniasis in Natal, Brazil. Transactions of the Royal Society of Tropical Medicine and Hygiene 88:386-388, 1994

16. Mestre GLC, Fontes CJF. A expansão da epidemia de leishmaniose visceral no estado do Mato Grosso, 1998-2005. Revista da Sociedade Brasileira de Medicina Tropical 40:42-48, 2007.

17. Ministério da Saúde. Manual de controle da leishmaniose visceral. Brasília, 2003.

18. Ministério da Saúde. Manual da leishmaniose visceral grave. Brasília, 2006.

19. Ministério da Saúde. Rede Interagencial de Informações para a Saúde. http:// tabnet.datasus.gov.br, Brasília, 2008.

20. Ministério da Saúde. Sistema de Informação de Agravos de Notificação. http:// tabnet.datasus.gov.br, Brasília, 2008.

21. Natal D. Fundamentos de epidemiologia. In: Phillippi Jr A, Romero MA, Bruna CG (eds) Curso de gestão ambiental. Editora Manole. São Paulo, p. 375-410, 2004
22. Oliveira AG, Andrade Filho JD, Falcão AL, Brazil RP. Estudo de flebotomíneos (Diptera Psychodidae, Phlebotominae) na zona urbana da cidade de Campo Grande, Mato Grosso do Sul, Brasil, 1999-2000. Cadernos de Saúde Pública 19:933-944, 2003.

23. Oliveira AG, Galati EAB, Oliveira 0, Oliveira GR, Espindola AIC, Dorval MEC, Brazil RP. Abundance of Lutzomyia longipalpis (Diptera: Psychodidae:Phlebotaminae) and urban transmission of visceral leishmaniasis in Campo Grande, state of Mato Grosso do Sul, Brazil. Memórias do Instituto Oswaldo Cruz 101:869-874, 2006

24. Queiroz MJA, Alves JGB, Correia JB. Leishmaniose visceral: características clínicoepidemiológicas em crianças de área endêmica. Jornal de Pediatria 80:141-146, 2004.

25. Rabello A, Orsini M, Dish J. Leishmania/HIV co-infection in Brazil: an appraisal. Annals of Tropical Medicine and Parasitology 97(supl I):17-28, 2003.

26. Salomon OD, Sinagra A, Nevot MC, Barberian G, Paulin P, Esteves JO, Riarte A, Estevez J. First visceral leishmaniasis focus in Argentina. Memórias do Instituto Oswaldo Cruz 103:109-111, 2008.

27. Silva EA, Andreotti R, Honer MR, Comportamento de Lutzomyia longipalpis, vetor principal da leishmaniose visceral americana em Campo Grande, estado do Mato Grosso do Sul. Revista da Sociedade Brasileira de Medicina Tropical 40:420-425, 2007.

28. Silva ES, Gontijo CMF, Pacheco RS, Fuiza VOP, Brazil RP. Visceral leishmaniasis in the metropolitan region of Belo Horizonte, state of Minas Gerais, Brazil. Memórias do Instituto Oswaldo Cruz 96:285-291, 2001.

29. Sherlock I. Ecological interactions of visceral leishmaniasis in state of Bahia, Brazil. Memórias do Instituto Oswaldo Cruz 91:671-683, 1996.

30. World Health Organization. Weekly Epidemiological Report 44:365-372, 2002

31. World Health Organization. Tropical Disease Research. Seventeenth Program Report. Progress 2003-2004. Geneva, 2005

32. World Health Organization. Report of the fifth consultative meeting on Leishmania/HIV co-infection. Addis Ababa, Ethiopia, 2007. 